London, England, 1965), p. 524.

2J. F. Perkins, Phys. Rev. 173, 164 (1968).

${ }^{3}$ I. Cheshire (private communication).

${ }^{4}$ A. Temkin, Phys. Rev. 107, 1004 (1957).

${ }^{5}$ M. H. Mittleman, Phys. Rev. A 2, 1846 (1970).

${ }^{6}$ C. J. Joachain and M. H. Mittleman, Phys. Rev. A 4, 1492 (1971).

${ }^{7}$ H. Feshbach, Ann. Phys. (N.Y.) 19, 287 (1962).

${ }^{8}$ M. H. Mittleman and K. M. Watson, Phys. Rev. $\underline{113}$,
198 (1959).

${ }^{9}$ E. Kujawski, Phys. Rev. A $\underline{5}, 2690$ (1972).

${ }^{10}$ See, for example, J. O. Hirschfelder, C. F. Curtiss, and R. B. Bird, Molecular Theory of Gases and Liquids (Wiley, New York, 1954), p. 952.

${ }^{11}$ E. Clementi, IBM J. Res. Develop. Suppl. 9, 2 (1965).

${ }^{12} \mathrm{I}$ am indebted to Dr. H. Tai for performing this calculation.

PHYSICAL REVIEW A

\title{
Hydrogenic Transitions in Multiply Charged Fe and Ni Ions*
}

\author{
W. N. Lennard, R. M. Sills, and W. Whaling \\ California Institute of Technology, Pasadena, California 91109 \\ (Received 1 May 1972)
}

\begin{abstract}
Ten lines in the range $3880 \leqq \lambda \leqq 5666 \AA$ in the beam-foil spectrum of iron have been identified with specific hydrogenic transitions in Fe IV-virl. The same transitions were observed from $\mathrm{Ni}$ and $\mathrm{Ar}$ beams. Deviations from the hydrogenic wavelengths are shown to be consistent with that expected from core polarization. The absence of these lines in astrophysical sources is discussed. A wavelength table is presented for identification of hydrogenic transitions to be expected in beam-foil spectra.
\end{abstract}

\section{INTRODUCTION}

In the visible radiation from a beam of iron ions excited by passage through a thin foil, Whaling et al. ${ }^{1}$ found that the strongest lines in their spectrograms had wavelengths not previously reported for any iron ion. In this paper we describe a measurement of the charge of the ions radiating the ten strongest visible lines from a 1. 5-MeV Fe ion beam. We propose transitions that will account for all of these wavelengths, and suggest that these same wavelengths will be seen in other heavy-ion beams when excited by the beam-foil method.

\section{EXPERIMENTAL METHODS}

The charge of the ion radiating the unknown wavelength was determined by measuring the displacement of the beam in a transverse electric field. An $\mathrm{Fe}^{+}$beam was accelerated to $1.3-\mathrm{MeV}$ energy and then further ionized and excited by passage through a thin $\left(10-\mu \mathrm{g} / \mathrm{cm}^{2}\right)$ carbon foil. Collimating slits $(0.25 \times 8 \mathrm{~mm})$ located both ahead of and behind the foil defined a narrow ribbon beam. This radiant beam was deflected normal to its wide dimension in a transverse electrostatic field extending $10 \mathrm{~cm}$ downstream from the foil.

A monochromator with a $10-\AA$ passband was placed to accept radiation from a $1-\mathrm{cm}$ length of an arbitrarily selected beam trajectory in the deflecting field. Radiation from any other trajectory was blocked by a mask. A narrow $(1 \mathrm{~cm} \times 1 \mathrm{~mm})$ slit in the mask, tangent to the selected trajectory, permitted radiation from the beam to reach the monochromator only if the beam was deflected along the selected trajectory. The deflecting voltage was varied to sweep the radiant beam across the slit. The product (deflecting voltage) $\times$ (ion charge)/(ion energy) is constant for a particular trajectory, and the value of this constant for the selected trajectory was determined by observing the voltage required to deflect a $0.65-\mathrm{MeV} \mathrm{N}^{2+}$ $(\lambda 4379)$ beam along this trajectory.

\section{RESULTS}

In Table I we present the charges measured for the ten strongest lines in the visible spectrum of the $1.5-\mathrm{MeV}$ iron ion beam. The wavelengths listed in the first column were measured from the iron spectrogram in Fig. 1. In scanning these lines with the monochromator preparatory to measuring the ion charge, it was found that most of the lines are composed of two or more components with a spacing of a few $\AA$. The wavelengths measured from the spectrogram are therefore an average over the several unresolved components. We estimate an uncertainty of $\pm 2 \AA$ in this average wavelength, even though the components may be spread over several $\AA$ about this average.

The measured charge is listed in the third column. The low efficiency of our photon detector (EMI 6256S) for wavelengths beyond $6000 \AA$ made it impossible to measure the charges of the last four lines in the table, and the assignments of an ionic charge to these transitions are based on the analysis described below.

Once the charges were known, it was observed 


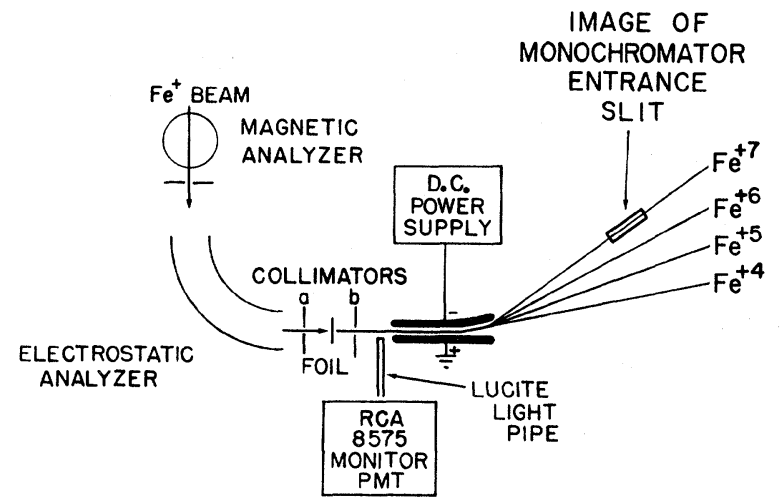

FIG. 1. Schematic diagram of the charge-deflection apparatus. The monochromator is positioned to receive light emitted out of the plane of the paper. Only light emitted from the 1-cm segment of the trajectory labeled "image of monochromator entrance slit" can reach the entrance slits of the monochromator. The deflection of the various charge states is greatly exaggerated in this figure: An actual off-axis deflection was $2 \mathrm{~mm}$.

that the measured wavelengths were very close to those for $\Delta n=1$ and $\Delta n=2$ transitions between states of a single electron moving in a hydrogenlike orbit around a central charge $(Q+1) e^{+}$, where $Q e^{+}$ is the ion charge. In column 4 we list the wavelengths computed from the hydrogenic terms $T$ $=-R(Q+1)^{2} / n^{2}$ for transitions between states with the principal quantum numbers listed in column 6 . The last four wavelengths in column 1 and $\lambda 4658$ were then classified by their near coincidence with

TABLE I. Identification of transitions in the $\mathrm{Fe}$ and $\mathrm{Ni}$ beam-foil spectra. The wavelengths are measured from spectrograms of Fig. 1 by comparison with an $\mathrm{Fe}$ reference spectrum.

\begin{tabular}{|c|c|c|c|c|c|}
\hline $\begin{array}{l}\mathrm{Fe} \\
(\AA)\end{array}$ & $\begin{array}{r}\mathrm{Ni} \\
(\AA)\end{array}$ & $\begin{array}{c}\text { Measured } \\
\text { Ion } \\
\text { Charge }\end{array}$ & $\begin{array}{c}\lambda_{\text {calc }} \\
(\AA)\end{array}$ & $\begin{array}{c}\text { Ion } \\
\text { Charge }\end{array}$ & $\Delta n$ \\
\hline 3879 & 3883 & $6+$ & 3888 & $6+$ & $8 \rightarrow 7$ \\
\hline 4336 & 4338 & $7+$ & 4342 & $7+$ & $9 \rightarrow 8$ \\
\hline 4497 & 4497 & $5+$ & 4500 & $5+$ & $10 \rightarrow 8$ \\
\hline 4516 & 4515 & $4+$ & 4521 & $4+$ & $9 \rightarrow 7$ \\
\hline 4553 & 4553 & $6+$ & 4557 & $6+$ & $11 \rightarrow 9$ \\
\hline 4625 & 4630 & $3+$ & 4660 & $3+$ & $6 \rightarrow 5$ \\
\hline 4658 & 4658 & $\ldots$ & 4660 & $7+$ & $12 \rightarrow 10$ \\
\hline 4680 & 4682 & $3+$ or $4+$ & 4687 & $3+$ & $8 \rightarrow 6$ \\
\hline 4903 & 4904 & $\ldots$ & 4946 & $4+$ & $7 h \rightarrow 6 g$ \\
\hline 4934 & 4935 & $4+$ & 4946 & $4+$ & $7 i \rightarrow 6 h$ \\
\hline 5283 & 5286 & $5+$ & 5292 & $5+$ & $8 \rightarrow 7$ \\
\hline 5665 & 5666 & $6+$ & 5671 & $6+$ & $9 \rightarrow 8$ \\
\hline 6063 & 6065 & $\ldots$ & 6070 & $7+$ & $10 \rightarrow 9$ \\
\hline 6079 & 6079 & $\ldots$ & 6086 & $6+$ & $12 \rightarrow 10$ \\
\hline 6197 & 6197 & $\ldots$ & 6202 & $5+$ & $11 \rightarrow 9$ \\
\hline 6475 & 6474 & $\ldots$ & 6480 & $4+$ & $10 \rightarrow 8$ \\
\hline
\end{tabular}

transitions expected for ions with charge $3+$ to $7+$ the major components of our beam. The wavelengths listed in column 4 include all of the $\Delta n=1$ and 2 transitions in our wavelength range $3500 \leq \lambda$ $\leq 6500 \AA$ and charges $3+\leq Q \leq 7+$.

To confirm our identification, we substituted a $\mathrm{Ni}$ beam for the Fe beam and repeated our measurements. The Ni spectrum is shown in the upper-half of Fig. 1. The striking similarity between the spectra of two different elements is convincing proof of the origin of these lines in common hydrogenic levels. We have seen the same lines from an argon beam, and we should expect to see the same lines in any ion beam containing ions of the same charge. Wavelengths measured from the Ni spectrogram appear in the second column of Table I.

The one-electron states excited in our experiment are expected to be more tightly bound than the Rydberg formula predicts for a one-electron ion because of (a) polarization of the ion core by the outer electron, (b) penetration of the core by the outer electron, and (c) the spin-orbit interaction. All of these interactions increase the electron binding and depend on $n$ and $l$ in a way that will shift transitions in which $n$ and $l$ decrease to the blue of the hydrogenic value, as observed in our spectra. According to Edlén, ${ }^{2}$ the polarization effect is much the largest of these three corrections to the binding energy for states with large $n$

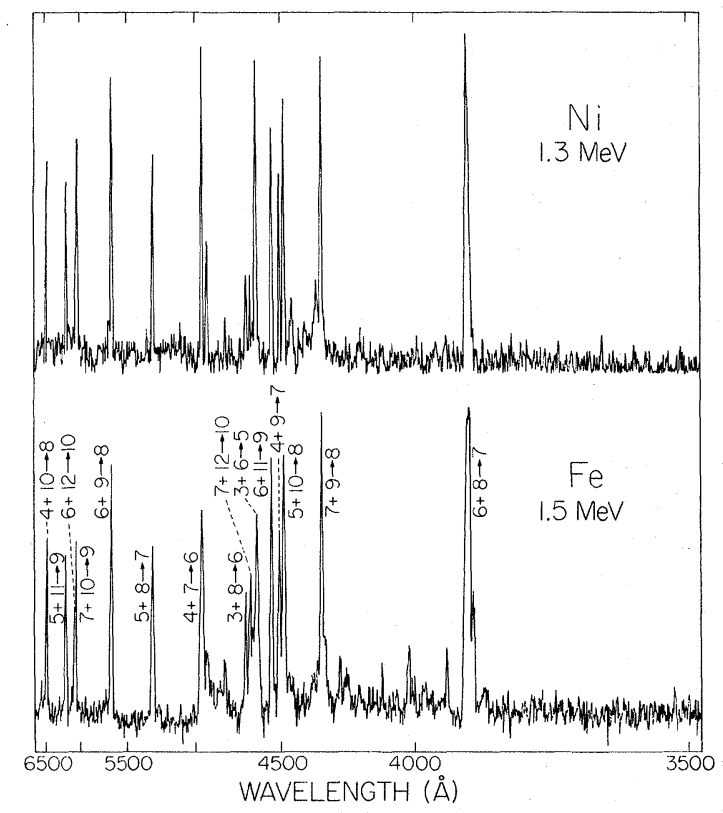

FIG. 2. Densitometer tracings of spectrograms of Ni and $\mathrm{Fe}$ beams excited by passage through $10-\mu \mathrm{g} / \mathrm{cm}^{2}$ carbon foils. The angle of observation is $90^{\circ}$. The Fe spectrum is reproduced from Ref. 1 , in which details of the exposure may be found. 
and $l$. Edlen gives formulas for computing the wavelength shift due to polarization, but these depend on the polarizability of the core which is in general not known. For large $n$ and $l$, the polarization correction approaches zero and the term values approach those given by the Rydberg formula.

The polarization of the core removes the degeneracy between states of the same $n$ but different $l$ and splits the hydrogenic lines into several components, and one expects the lines to exhibit a multiplet structure as well as a shift to the blue. For most of the transitions that we observed, this structure is too close to be evident in the spectrograms of Fig. 1. As mentioned above, many of the lines that appear single in the spectrograms have been found to have two or more components, separated by a few $\AA$, but our measurements are not sufficiently precise to permit a detailed comparison of the observed structure and theoretical predictions. The only evidence for this splitting apparent in Fig. 1 is the weak line at $4903 \AA$ which may correspond to the $7 h-6 g$ satellite of the $7 i-6 h$ line at $4935 \AA$. This weak line appears in both the Fe and Ni spectra, and there are no other likely hydrogenic transitions close to the observed wavelength. If one uses the observed shift of the 4935- $\AA$ line to compute a dipole polarizability for the $\mathrm{Fe}^{5+}$ core, one can then compute the expected position of the $7 h \rightarrow 6 g$ component. In this way, we find $\alpha_{a}\left(\mathrm{Fe}^{5+}\right)=1.0 a_{0}^{3}$ and compute $\lambda(7 h-6 g)=4906$ $\AA$, close to the observed value of $4903 \AA$. Supporting this interpretation is the fact that one expects the reddest component of the multiplet to be strongest, since states with maximum $l=n-1$ are favored as the electron cascades down from states with high- $n$ values. One feature that is not accounted for in this analysis is the fact that $\lambda 4935$ is actually a doublet, with two nearly equal components separated by $6 \AA$. This spacing is too large to be accounted for by spin-orbit splitting and must reflect some coupling of the outer electron with the $\mathrm{Fe}^{5+}$ core.

\section{DISCUSSION}

Hydrogenic transitions between states of large $n$ in multiply charged ions have been reported in many beam-foil (BF) spectra, but they are rarely observed in other laboratory sources and not at all in astrophysical sources. Gabriel ${ }^{3}$ has observed such transitions in a high-density plasma pinch, and in particular has observed $\lambda 5280$ in $\mathrm{O}^{5+}$, the same line we have seen with $\mathrm{Fe}, \mathrm{Ni}$, and $\mathrm{Ar}$ beams. This same transition and several others between high- $n$ levels have been reported from $\mathrm{C}^{5+}$ in the plasma generated when a high-intensity laser beam strikes a solid surface. ${ }^{4}$ However, there is no other light source in which hydrogenic transitions are so prominent as they are in Fig. 1 , and only in BF spectra have they been observed with ions as heavy as $\mathrm{Fe}$ or $\mathrm{Ni}$.

It is interesting to inquire why these states are so readily excited in the $\mathrm{BF}$ interactions, and why they are seldom seen in other sources. Turning to the latter question first, their high excitation energy, hundreds of $\mathrm{eV}$, is responsible for their weakness in a low-density plasma such as the solar corona, where Huang ${ }^{5}$ has shown that excitation by electron capture is negligible compared to excitation by collision. The upper levels of identified coronal lines are typically at only a few eV of excitation energy. Dalgarno ${ }^{6}$ has pointed out that the relatively long radiative lifetime of the hydrogenic states, increasing roughly as $n^{4.5}$, combined with their large cross section for collisional destruction, account for their weakness in a local thermodynamic equilibrium (LTE) plasma.

The same argument explains their absence in beams excited by passage through a gas. Brown et $a l .{ }^{7}$ have compared the radiation from a 900 keV sodium beam excited by passage through a carbon foil with the radiation from the same beam passing through helium at a pressure of 0.1 torr. The strongest line observed in the BF spectrum, $\lambda 4649$, was not seen at all in the gas-excited spectrum. The charge of the ion radiating this strong line was measured by Brown, and later by Dufay et al.,$^{8}$ to be $3+$. The $n=6-5$ transition in the $3+$ ion is at $4660 \AA$. From our estimate of the dipole polarizability of the seven-electron core, $\alpha_{d}=0.51 a_{0},{ }^{3}$ we would expect a wavelength shift of $-12 \AA$, in good agreement with the observed shift of $-11 \AA$.

The absence of this line in Brown's gas-excited spectrum can be understood by comparing the mean time between collisions, $1.5 \times 10^{-10} \mathrm{sec}$, on the assumption of $\sigma_{\text {col1 }}=\pi\left(n^{2} a_{0} / Z\right)^{2}$, with the radiative lifetime of the hydrogenic state, $24 \times 10^{-10}$ sec. ${ }^{9}$ The large collision cross section favors nonradiative deexcitation of the state. It would be of interest to repeat Brown's experiment with the gas pressure reduced by a factor of 100 to see if the hydrogenic line appears.

The prominence of hydrogenic transitions in BF spectra suggests that electron capture makes an important contribution to the excitation of the beam. In the usual picture of the passage of a charged particle through matter, the moving ion continually loses and recaptures its more loosely bound electrons in repeated encounters with the atoms of the absorber. The charge on the ion emerging from the surface is the net result of this sequence of many capture and loss events. The excitation of the emerging ion is brought about either by the last capture into an excited state, or by the last collision before the ion emerges into 
TABLE II. Hydrogenic wavelengths: $\lambda^{-1}=R(Q+1)^{2}\left[1 / n^{2}-1 /(n+\Delta n)^{2}\right]$. Values in the table are for mass $A=46, R$ $=109736 \mathrm{~cm}^{-1}$. For other masses, $\lambda_{A}=\lambda_{46}\left[1+1.2 \times 10^{-5}(46-A) / A\right]$. Table includes all transitions with $\Delta n=1,2$, or 3 , in the wavelength range $1000-8000 \AA$.

\begin{tabular}{|c|c|c|c|c|c|c|c|c|c|c|c|c|c|c|}
\hline Ion & $n$ & $(n+1)$ & $(n+2)$ & $(n+3)$ & Ion & $n$ & $(n+1)$ & $(n+2)$ & $(n+3)$ & Ion & $n$ & $(n+1)$ & $(n+2)$ & $(n+3)$ \\
\hline \multirow[t]{3}{*}{$X^{+}$} & 2 & 1640 & 1215 & 1085 & \multirow[t]{9}{*}{$X^{8+}$} & 6 & 1527 & 926 & 729 & \multirow[t]{13}{*}{$X^{12+}$} & 7 & 1127 & 669 & 518 \\
\hline & 3 & 4687 & 3204 & 2734 & & 7 & 2352 & 1395 & 1081 & & 8 & 1644 & 959 & 733 \\
\hline & 4 & 10125 & 6561 & 5412 & & 8 & 3431 & 2000 & 1528 & & 9 & 2299 & 1321 & 998 \\
\hline \multirow[t]{4}{*}{$X^{2+}$} & 3 & 2083 & 1424 & 1215 & & 9 & 4796 & 2757 & 2083 & & 10 & 3107 & 1765 & 1321 \\
\hline & 4 & 4500 & 2916 & 2406 & & 10 & 6482 & 3682 & 2756 & & 11 & 4085 & 2297 & 1705 \\
\hline & 5 & 8284 & 5168 & 4154 & & 11 & 8523 & 4793 & 3558 & & 12 & 5249 & 2927 & 2157 \\
\hline & 6 & 13739 & 8332 & 6561 & & 12 & 10952 & 6106 & 4500 & & 13 & 6615 & 3661 & 2681 \\
\hline \multirow{7}{*}{$X^{3+}$} & 3 & 1172 & 801 & 683 & & 13 & 13802 & 7639 & 5595 & & 14 & 8200 & 4509 & 3284 \\
\hline & 4 & 2531 & $\begin{array}{r}001 \\
1640\end{array}$ & 683 & & 14 & 17108 & 9408 & 6852 & & 15 & 10019 & 5479 & 3971 \\
\hline & 4 & $\begin{array}{l}2531 \\
4660\end{array}$ & $\begin{array}{l}1640 \\
2907\end{array}$ & 1353 & \multirow[t]{10}{*}{$X^{9+}$} & 6 & 1237 & 750 & 591 & & 16 & 12089 & 6577 & 4746 \\
\hline & 5 & $\begin{array}{l}4660 \\
7728\end{array}$ & $\begin{array}{l}2907 \\
4687\end{array}$ & 2337 & & 7 & 1905 & 1130 & 876 & & 17 & 14426 & 7813 & 5616 \\
\hline & 6 & $\begin{array}{r}7728 \\
11907\end{array}$ & 4687 & 3691 & & 8 & 2779 & 1620 & 1238 & & 18 & 17046 & 9195 & 6585 \\
\hline & 7 & 11907 & 7064 & 5472 & & 9 & 3885 & 2233 & 1687 & & 19 & 19965 & 10731 & 7660 \\
\hline & 8 & 17368 & 10125 & 7738 & & 10 & 5251 & 2982 & 2232 & \multirow{13}{*}{$X^{13+}$} & 8 & 1418 & 827 & 632 \\
\hline \multirow[t]{6}{*}{$X^{4+}$} & 4 & 1620 & 1050 & 866 & & 11 & 6904 & 3882 & 2882 & & 9 & 1982 & 1139 & 861 \\
\hline & 5 & 2982 & 1861 & 1495 & & 12 & 8871 & 4946 & 3645 & & 10 & 2679 & 1522 & 1139 \\
\hline & 6 & 4946 & 2999 & 2362 & & 13 & 11180 & 6188 & 4532 & & 11 & 3522 & 1981 & 1470 \\
\hline & 7 & 7621 & 4521 & 3502 & & 14 & 13858 & 7621 & 5550 & & 12 & 4526 & 2524 & 1860 \\
\hline & 8 & 11115 & 6480 & 4952 & & 15 & 16932 & 9259 & 6710 & & 13 & 5704 & 3157 & 2312 \\
\hline & 9 & 15540 & 8931 & 6749 & \multirow{12}{*}{$X^{10+}$} & 6 & 1022 & 620 & 488 & & 14 & 7070 & 3888 & 2832 \\
\hline \multirow[t]{7}{*}{$X^{5^{+}}$} & 4 & 1125 & 729 & 601 & & 7 & 1575 & 934 & $\begin{array}{l}720 \\
724\end{array}$ & & 15 & 8639 & 4724 & 3424 \\
\hline & 5 & 2071 & 1292 & 1038 & & 8 & 2297 & 1339 & 1023 & & 16 & 10424 & 5671 & 4092 \\
\hline & 6 & 3435 & 2083 & 1640 & & 9 & 3211 & 1845 & 1394 & & 17 & 12439 & 6737 & 4842 \\
\hline & 7 & 5292 & 3140 & 2432 & & 10 & 4339 & 2465 & 1845 & & 18 & 14698 & 7928 & 5678 \\
\hline & 8 & 7719 & 4500 & 3439 & & 11 & 5705 & 3208 & 2381 & & 19 & 17215 & 9252 & 6605 \\
\hline & 9 & 10791 & 6202 & 4687 & & 12 & 7331 & 4088 & 3012 & & 20 & 20004 & 10716 & 7626 \\
\hline & 10 & 14585 & 8284 & 6200 & & 13 & 9239 & 5114 & 3745 & \multirow[t]{19}{*}{$X^{14^{+}}$} & 8 & 1235 & 720 & 550 \\
\hline \multirow{8}{*}{$X^{6^{+}}$} & 5 & 1522 & 949 & 763 & & 14 & 11453 & 6298 & 4587 & & 9 & 1727 & 992 & 750 \\
\hline & 6 & 2524 & 1530 & 1205 & & 15 & 13993 & 7652 & 5546 & & 10 & 2334 & 1325 & 992 \\
\hline & 7 & 3888 & 2307 & 1787 & & 16 & 16885 & 9186 & 6629 & & 11 & 3068 & 1725 & 1281 \\
\hline & 8 & 5671 & 3306 & 2527 & & 17 & 20148 & 10913 & 7843 & & 12 & 3943 & 2198 & 1620 \\
\hline & 9 & 7928 & 4557 & 3443 & \multirow[t]{14}{*}{$X^{11^{+}}$} & 7 & 1323 & 785 & 608 & & 13 & 4969 & 2750 & 2014 \\
\hline & 10 & 10716 & 6086 & 4555 & & 8 & 1930 & 1125 & 860 & & 14 & 6159 & 3387 & 2467 \\
\hline & 11 & 14089 & 7923 & 5881 & & 9 & 2698 & 1551 & 1172 & & 15 & 7525 & 4115 & 2982 \\
\hline & 12 & 18104 & 10094 & 7439 & & 10 & 3646 & 2071 & 1550 & & 16 & 9080 & 4940 & 3565 \\
\hline \multirow{10}{*}{$X^{7+}$} & & & & & & 11 & 4794 & 2696 & 2001 & & 17 & 10835 & 5869 & 4218 \\
\hline & $\begin{array}{l}5 \\
6\end{array}$ & $\begin{array}{l}1165 \\
1932\end{array}$ & $\begin{array}{r}727 \\
1172\end{array}$ & $\begin{array}{l}584 \\
923\end{array}$ & & 12 & 6160 & 3435 & 2531 & & 18 & 12803 & 6907 & 4946 \\
\hline & 6 & 1932 & 1172 & $\begin{array}{r}923 \\
1368\end{array}$ & & 13 & 7764 & 4297 & 3147 & & 19 & 14996 & 8060 & 5753 \\
\hline & 7 & 2977 & 1766 & 1368 & & 14 & 9623 & 5292 & 3854 & & 20 & 17425 & 9335 & 6643 \\
\hline & 8 & 4342 & 2531 & 1934 & & 15 & 11758 & 6430 & 4660 & & 21 & 20104 & 10737 & 7621 \\
\hline & 9 & 6070 & 3489 & 2636 & & 16 & 14188 & 7719 & 5570 & & & & & \\
\hline & 10 & 8204 & 4660 & 3487 & & 17 & 16930 & 9170 & 6591 & & & & & \\
\hline & 11 & 10787 & 6066 & 4502 & & 18 & 20005 & 10791 & 7728 & & & & & \\
\hline & 12 & 13861 & 7728 & 5695 & & & & & & & & & & \\
\hline & 13 & 17468 & 9668 & 7081 & & & & & & & & & & \\
\hline
\end{tabular}

the vacuum.

The excited levels observed in this experiment have very large mean radii, $10-20 \AA$, whereas the mean spacing between atoms of the foil is $\sim 2$ $\AA$. They have excitation energies of several hundred $\mathrm{eV}$, whereas the electron energy at the ion velocity is only $14 \mathrm{eV}$. It seems clear that these levels are populated by the last capture event at the surface, not by collisional excitation. There is a high electron density at the surface, and the surface can absorb energy to permit nonradiative capture.

Different models of electron capture at a surface lead to different distributions of population of the excited levels. (a) If one assumes, following Bohr, ${ }^{10}$ that the states favored in the capture process are those for which the electron velocity [ $2 \times$ (binding energy) $\left./ m_{0}\right]^{1 / 2}$ is close to the velocity of the moving ion, one expects an ion of charge $Q$ to show large initial population in states with 
$n \approx(Q+1)\left[24.8 A_{\text {ion }} / E_{\mathrm{ion}}(\mathrm{keV})\right]^{1 / 2}$. (b) If one adopts Oliphant and Moon's ${ }^{11}$ assumption that capture is favored into states bound to the ion by an amount close to the work function of the surface, one would expect the primary population to be concentrated in states with $n \sim 1.7(Q+1)$, assuming a work function of $4.6 \mathrm{eV}$ for the $C$ surface. (c) Free-electron recombination that takes place after the ion has escaped from the influence of the surface would lead to an initial population that decreases with increasing excitation energy as $1 / n^{3}$. We have examined our own results and the published observations of others and conclude that the third assumption is favored by the meager information presently available. The pertinent observations are these:

(i) The levels that are observed are limited only by the wavelength range of the experiment. For the ions which compose the major components of the beam, every $\Delta n=1$ and 2 transition lying within the wavelength range of the detector is observed. There seems to be no upper bound on the excitation energy of the initial states populated.

(ii) For a given ion, transitions from levels of lower $n$ appear to be stronger. Cascading contributes to this behavior and obscures the initial population.

(iii) Light decay curves for the level with quantum number $n_{u}$ typically indicate cascading into the upper level from still higher levels with $n \geqq n_{u}$ +1 , consistent with our conclusion from observation (i). However, there are no observations of increasing decay curves that would indicate a population in the $n_{u}+1$ level much greater than that in the $n_{u}$ level. Observations (ii) and (iii) suggest an initial population that is either independent of $n$ or decreases with increasing $n$.

One is led to the conclusion that only assumption (c) is consistent with the observations. However, it is surprising that levels with $n \sim 10$ are seen at all if the population falls off as rapidly as $n^{-3}$. Jordan et al. ${ }^{12}$ observed a population in $\mathrm{He}^{+}$that falls off more rapidly than $n^{-3}$, but it has been suggested that the behavior of helium and of heavy ions are qualitatively different. Dmitriev et al. ${ }^{13} \mathrm{re}-$ port that "electron capture by ions with $Z \geqq 4$ (unlike protons) occurs mainly in excited states." Further study of the initial populations should provide a valuable insight into the excitation process.

In conclusion, it should be noted that observation (i) above should aid experimenters in identifying unknown lines in their spectra. Hydrogenic lines appear in many published spectra without identification. As an aid to identification we list in Table II the wavelengths computed from the Rydberg formula for ions of charge $1^{+}-14^{+}$. We list transitions for $\Delta n=1,2$, and 3 , although $\Delta n=3$ transitions in heavy ions are rare. ${ }^{14}$

\section{ACKNOWLEDGMENTS}

The authors are indebted to Alan Gabriel, L. C. Cocke, and S. Bashkin for helpful disucssions and access to unpublished results.
* Supported in part by NSF Grant No. GP-28027 and the Office of Naval Research Grant No. N00014-67-A0094-0022.

${ }^{1}$ W. Whaling, R. B. King, and P. L. Smith, in BeamFoil Spectroscopy, edited by S. Bashkin (Gordon and Breach, London, 1968), Vol. I. p. 81.

${ }^{2}$ B. Edlén, in Handbüch der Physik, edited by S. Flügge (Springer-Verlag, Berlin, 1964), Vol. 27, p. 80 .

${ }^{3}$ A. H. Gabriel, in Proceedings of the Fourth International Conference on Ionization Phenomena in Gases, edited by N. R. Nilsson (North-Holland, Amsterdam, $1960)$, p. 829 .

${ }^{4}$ B. C. Boland, F. E. Irons, and R. W. P. McWhirter, J. Phys. B 1, 1180 (1968).

${ }^{5} \mathrm{~K}$. Huang, Astrophys. J. 101, 187 (1945).

${ }^{6} \mathrm{~A}$. Dalgarno, in Proceedings of the First International Conf evence on Atomic Physics, edited by B. Bederson (Plenum, New York, 1969), p. 169.
${ }^{7}$ L. Brown, W. K. Ford, V. Rubin, W. Trachslin, and W. Brandt, in Ref. 1, p. 45.

${ }^{8}$ M. Dufay, M. Gaillard, and S. M. Carré, Phys. Rev. A 3,1367 (1971).

${ }^{9}$ H. A. Bethe and E. E. Salpeter, in Ref. 2, Vol. 35, p. 88 .

${ }^{10}$ N. Bohr, Phys。 Rev. 59, 270 (1941).

${ }^{11}$ M. L. E. Oliphant and P. B. Moon, Proc. Roy. Soc. (London) A127, 388 (1930).

${ }^{12}$ J. A. Jordan, Jr., G. S. Bakken, and R. E. Yager, J. Opt. Soc. Am. 57, 530 (1967).

${ }^{13}$ I. S. Dmitriev, Y. A. Teplova, and V. S. Nikolaev, Zh. Eksperim. i Teor. Fiz. 61, 1359 (1971) [Sov. Phys. JETP 34, 723 (1972)].

${ }^{14}$ R. Hallin, J. Lindskog, A. Marelius, J. Pihl, and R. Sjodin, in Proceedings of the Second European Conference on Beam-Foil Spectroscopy, Lyon, 1971 (unpublished). 